\title{
Culture Supernatants from Lactobacillus plantarum Induce Necrosis on a Human Promyelocytic Leukemia Cell Line
}

\author{
M. A. Puertollano, E. Puertollano, G. Álvarez de Cienfuegos and M. A. de Pablo \\ Division of Microbiology, Department of Health Sciencies, University of Jaén, Spain
}

\begin{abstract}
Lactic acid bacteria (LAB) and bioactive peptides from these LAB are known to have antitumoral properties ${ }^{(1)}$, although the intrinsic mechanisms responsible for the execution of this activity are poorly understood. Previous work in our laboratory reported that concentrated supernatants from $L$. plantarum reduce the development of HL-60 cells and increase the erythrocyte hemolysis ${ }^{(2)}$. Here we investigated the ability of concentrated supernatants from $L$. plantarum to promote cell death in this human promyelocytic cell line. This study was aimed to test the hypothesis that concentrated supernatants from L. plantarum at 5,50 or $100 \mu \mathrm{g} / \mathrm{ml}$ for $24 \mathrm{~h}$ exert cytotoxic effects on HL-60 cells. Accumulation of reactive oxygen species (ROS) was diminished and nuclear staining with Hoechst 33342 and propidium iodide (PI) determined a necrotic induction in a concentration-dependent sequence. Concentrated supernatants did not modify or reduced the activity of caspase-3. The assessment of phosphatidylserine externalization by annexin V/PI double staining led to a necrotic state, but the treatment did not produce a dissipation of mitochondrial membrane potential $(\Delta \psi \mathrm{m})$, whereas cell cycle analysis revealed that concentrated supernatants failed to significantly enhance the population of HL-60 cells in the hypodiploid (sub-G1) fraction. In this study we demonstrated the cytotoxic properties of concentrated supernatants from L. plantarum on a tumour cell line, and then, to open the possibility to analyze the chemical composition to elucidate the bioactive molecules.
\end{abstract}

1. LeBlanc JG, Matar C, Valdez JC, LeBlanc J \& Perdigon G (2002) J Dairy Sci 5, 2733-2742.

2. Puertollano E, Puertollano MA, Cruz-Chamorro L, Álvarez de Cienfuegos G, Ruiz-Bravo A \& de Pablo MA (2009) J Appl Microbiol 106, 1194-1203. 